\title{
The Sagittal Balance of the Cervical Spine: Radiographic Analysis of Interdependence between the Occipitocervical and Spinopelvic Alignment
}

\author{
Babak Alijani, Javid Rasoulian \\ Department of Neurosurgery, Poorsina Hospital, School of Medicine, Guilan University of Medical Science, Rasht, Iran
}

Study Design: This was a prospective clinical study.

Purpose: Previous studies have indicated that cervical lordosis is a parameter influenced by segmental and global spinal sagittal balance parameters. However, this correlation still remains unclear. Therefore, a better understanding of the normal values and interdependencies between inter-segmental alignment parameters is needed. This is a preliminary analysis that helps to understand these factors.

Overview of Literature: Change in global sagittal alignment is associated with poor health-related quality of life. Questions regarding which parameters play the primary roles in the progression of spinal sagittal imbalance and which might be compensatory factors remain unanswered.

Methods: Prospectively, 420 adults (105 asymptomatic, 105 cervical symptomatic, 105 lumbar symptomatic, and 105 post-surgical) were selected. Whole-spine standing lateral radiographs were taken, and spinopelvic, thoracic, and cervical parameters were measured. Then, the data were analyzed using correlation coefficient test and multiple regression analysis.

Results: All the parameters showed a normal distribution. The mean values of the cervical parameters are as follows: $C_{1} C_{2}$ Cobb angle, $-27.07^{\circ} \pm 4.3^{\circ} ; C_{2} C_{7}$ Cobb angle, $-16.4^{\circ} \pm 5.6^{\circ} ; O_{C} C_{2}$ Cobb angle, $-14.5^{\circ} \pm 3.8^{\circ} ; O_{C} C_{7}$ Cobb angle, $-29.8^{\circ} \pm 5.6^{\circ} ; C_{2} C_{7}$ Harrison angle, $20.4^{\circ} \pm 4.3^{\circ}$; and $C_{7}$ slope, $-25.4^{\circ} \pm 5.6^{\circ}$. The analysis of these parameters revealed no statistically significant difference between asymptomatic, symptomatic, and post-surgical patients. $C_{7}$ sagittal vertical axis (SVA) correlated with the $C_{2} C_{7}$ Cobb angle $(r=0.7)$ in all groups. No significant correlation was noted between cervical and spinopelvic parameters in asymptomatic patients. However, $C_{1} C_{2}$ Cobb angle correlated significantly with pelvic incidence $(\mathrm{PI}, r=-0.2)$, lumbar lordosis $(\mathrm{LL}, r=0.2)$, and pelvic tilt $(\mathrm{PT}, r=-0.2)$ in cervical symptomatic patients. Irrespective of the patient symptom sub-group $(\mathrm{n}=420), \mathrm{C}_{1} \mathrm{C}_{2} \mathrm{Cobb}$ angle correlated with $\mathrm{LL}(r=0.1)$ and $\mathrm{C}_{2} \mathrm{C}_{7} \mathrm{Har}$ rison angle correlated with $\mathrm{Pl}$ and $\mathrm{PT}(r=0.1)$.

Conclusions: Our results indicate significant interdependence between the spinopelvic and cervical alignment, especially in cervical symptomatic patients. In addition, strong correlation was found between the $\mathrm{C}_{7} \mathrm{SVA}$ and $\mathrm{C}_{2} \mathrm{C}_{7} \mathrm{Cobb}$ angle. Overall, the results of this study could help to better understand the cervical sagittal alignment and serve as preliminary data for planning surgical reconstruction procedures.

Keywords: Cervical spine; $\mathrm{C}_{7}$ slope; Cervical sagittal vertical axis; Spinopelvic sagittal balance; Alignment changes

Received May 18, 2019; Revised Jul 2, 2019; Accepted Jul 21, 2019

Corresponding author: Javid Rasoulian

Department of Neurosurgery, Poorsina Hospital, Rasht, Guilan, Iran

Tel: +98-1333368773, Fax: +98-1333311472, E-mail: javidrasoulian@gmail.com 


\section{Introduction}

The spine and the body function within a cone of equilibrium with focus in maintaining sagittal and coronal alignment with minimum energy expenditure. This happens with a harmonious relationship involving cervical lordosis (CL), thoracic kyphosis (TK), lumbar lordosis (LL), and pelvic anatomy. The purpose is mostly to maintain a mechanical balance in the sagittal and coronal planes centered from the center of the cranial mass, femoral heads, and lower extremities [1]. Many authors have reported that sagittal balance rather than coronal balance is significantly correlated with health-related quality of life, especially in patients who received surgical treatment [2-4]. Therefore, more attention is often paid to sagittal balance than coronal balance during spinal deformity assessment, surgical plan-making, and surgical procedure. Generally, sagittal imbalance results in increased muscular effort and energy expenditure, causing pain, fatigue, and disability [5]. Sagittal imbalance of the spine as a crucial factor in the pathogenesis of myelopathy is supported by several reports [6,7]. Multiple studies have described normative values for parameters of spinopelvic alignment in different populations of varying ages and pathologic conditions. In these studies, the interrelation of pelvic parameters, LL, TK, sagittal imbalance, and its effect on the quality of life and patient outcome has been well-established $[5,8,9]$. Many studies have reported that pelvic incidence (PI), a constant morphological parameter in each individual, has a significant influence on sagittal alignment of the lumbar spine or the thoracic spine, such as LL and TK [10]. The correlation between the cervical and thoracic spinal parameters is weaker than that between the lumbar and pelvic spinal parameters $[11,12]$. We started our study with the hypothesis that the overall sagittal balance of the thoracolumbar and lumbosacral spine is expected to influence the cervical spine, with the justification that the cervical spine is not an independent unit because it is connected to the thoracic spine. The aim of this study is to explore the correlation between the lumbosacral parameters and cervical sagittal balance, hoping that the results could provide guidance for changing these primary contributors in correction surgery.

\section{Materials and Methods}

\section{Materials}

The study was conducted prospectively over a period of 1 year in Guilan University of Medical Sciences (Poorsina Hospital) after obtaining approval from the institutional review board and ethics committee (approval no., IR.GUMS.REC.1396.278). Informed consent was obtained from all individual participants included in the study. A total of 420 adult volunteers were placed in four groups (each contains 105 subjects). Inclusion criteria for each group were as follows: group 1 (lumbar subjects) includes patients with lumbar pain or lower limb radiculopathy; group 2 (cervical subjects) includes patients with cervical pain or upper limb radiculopathy; group 3 (post-cervical surgery subjects) includes patients undergoing cervical discectomy and fusion or laminectomy/ laminoplasty without initial cervical deformity; and group 4 (normal subjects) includes asymptomatic volunteers without any complaints of low back, hip, or knee pain and no diagnosis or treatment related to the whole spine. The exclusion criteria were age lesser than 18 years, history of spinal trauma, history of bony diseases, and radiologic abnormalities, including degenerative changes, osteophyte formation, or global sagittal imbalance more than $5 \mathrm{~cm}$ from the sagittal vertical axis (SVA). A whole-spine lateral radiograph (91.44-cm cassettes) was obtained in a natural standing upright position with the head and trunk vertical and looking straight ahead and elbows fully flexed and fists resting on clavicles.

\section{Measurements}

All radiographical evaluation was performed digitally by two neurosurgery residents using the picture archiving and communication system. Measured radiographic parameters included the occipitocervical $\left(\mathrm{C}_{1} \mathrm{C}_{2}\right.$ Cobb angle, $\mathrm{O}_{\mathrm{C}} \mathrm{C}_{2}$ Cobb angle), cervical $\left(\mathrm{C}_{2} \mathrm{C}_{7}\right.$ Cobb angle, $\mathrm{C}_{2} \mathrm{C}_{7}$ Harrison angle, $\mathrm{O}_{\mathrm{C}} \mathrm{C}_{7} \mathrm{Cobb}$ angle, $\mathrm{C}_{7}$ slope), thoracic (thoracic inlet angle [TIA] and TK), spinopelvic (LL, sacral slope [SS], PI, and pelvic tilt [PT]), and $\mathrm{C}_{7}$ SVA. Definitions of the radiographic parameters are summarized in Table 1 [13].

All data were analyzed with the IBM SPSS ver. 24.0 software (IBM Corp., Armonk, NY, USA). D’Agostino kurtosis and skewness normality test was used to exam- 
Table 1. Definition of the radiographic parameters

\begin{tabular}{|c|c|c|}
\hline Parameter & Abbreviation & Definition \\
\hline $\mathrm{O}_{\mathrm{c}} \mathrm{C}_{2}$ Cobb angle & $\mathrm{O}_{\mathrm{c}} \mathrm{C}_{2 \mathrm{c}}$ & The angle between McRae line and the $\mathrm{C}_{2}$ lower end plate \\
\hline $\mathrm{O}_{\mathrm{c}} \mathrm{C}_{7}$ Cobb angle & $\mathrm{O}_{c} \mathrm{C}_{7 \mathrm{c}}$ & The angle between the McRae line and inferior endplate of $\mathrm{C}_{7}$ \\
\hline $\mathrm{C}_{1} \mathrm{C}_{2}$ Cobb angle & $\mathrm{C}_{1} \mathrm{C}_{2 \mathrm{c}}$ & The angle created by a line drawn parallel to the inferior aspect of $C_{1}$ and the line below $C_{2}$ \\
\hline $\mathrm{C}_{2} \mathrm{C}_{7}$ Cobb angle & $\mathrm{C}_{2} \mathrm{C}_{7 \mathrm{c}}$ & $\begin{array}{l}\text { The angle created by a line parallel to the inferior endplate of } C_{2} \text { and a line parallel to the inferior } \\
\text { endplate of } C_{7}\end{array}$ \\
\hline $\mathrm{C}_{2} \mathrm{C}_{7}$ Harrison angle & $\mathrm{C}_{2} \mathrm{C}_{7} \mathrm{H}$ & $\begin{array}{l}\text { The angle created by a line parallel to the posterior border of } C_{2} \text { and a line parallel to the posterior } \\
\text { border of } C_{7}\end{array}$ \\
\hline $\mathrm{C}_{7}$ slope & $\mathrm{C}_{7} \mathrm{~S}$ & The angle created by a line parallel to the superior endplate of $C_{7}$ and a horizontal reference line \\
\hline Thoracic inlet angle & $\mathrm{TIA}$ & $\begin{array}{l}\text { The angle created by a line from the center of the } T_{1} \text { UEP perpendicular to the } T_{1} \text { UEP and a line con- } \\
\text { necting the center of the } T_{1} \text { UEP and the upper end of the sternum }\end{array}$ \\
\hline Lumbar lordosis & $\mathrm{LL}$ & $\begin{array}{l}\text { The angle created by a line parallel to the superior endplate of } L_{1} \text { and a line parallel to the superior } \\
\text { endplate of } S_{1}\end{array}$ \\
\hline Pelvic incidence & $\mathrm{Pl}$ & $\begin{array}{l}\text { The angle created by a line drawn from the hip axis to the midpoint of the sacral end plate and a line } \\
\text { perpendicular to the center of the sacral end plate }\end{array}$ \\
\hline Sacral slope & SS & The angle created by a line parallel to the superior endplate of $S_{1}$ and a horizontal reference line \\
\hline Pelvic tilt & PT & $\begin{array}{l}\text { The angle created by a straight line connecting the midpoint of the bilateral femoral head center to } \\
\text { the midpoint of the sacral plate and the plumb line }\end{array}$ \\
\hline $\mathrm{C}_{7}$ sagittal vertical axis & C7SVA & $\begin{array}{l}\text { The horizontal distance between the postero-superior corner of the sacrum at } S_{1} \text { and a vertical plumb } \\
\text { line centered in the middle of the } C_{7} \text { vertebral body }\end{array}$ \\
\hline
\end{tabular}

UEP, upper-end plate.

Table 2. Demographic data of the study population

\begin{tabular}{|c|c|c|c|c|c|}
\hline Characteristic & Asymptomatic & Lumbar group & Cervical group & Post-surgical group & $p$-value \\
\hline Age (yr) & $58.55 \pm 7.5$ & $59.96 \pm 7.04$ & $57.8 \pm 7.94$ & $59.77 \pm 6.11$ & 0.095 \\
\hline Sex & & & & & 0.496 \\
\hline Male & 57 (54.3) & $59(56.2)$ & 58 (55.2) & $49(46.7)$ & \\
\hline Female & 48 (45.7) & 46 (43.8) & 47 (44.8) & $56(53.3)$ & \\
\hline
\end{tabular}

Values are presented as mean \pm standard deviation or number (\%).

ine whether the parameters were normally distributed. To test interobserver and intraobserver reliabilities, in 20 subjects, the parameters were measured at 2 separate times in 2-week interval and the interobserver and intraobserver intraclass correlation coefficients were analyzed by the mean measurement values of each observer. Descriptive data are given as the mean \pm standard deviation. Correlations between parameters were analyzed with the Pearson's correlation coefficient test, and linear regression models were set up. A p-value of less than 0.05 was considered significant.

\section{Results}

\section{Normality test}

The D'Agostino kurtosis and skewness normality test accepted the normality for all the spinal parameters, representing real-valued random variables. Histogram of normality of parameters is shown in Fig. 1.

\section{Demographic data}

Of the 420 subjects analyzed, $53.1 \%$ were males and $46.9 \%$ were females with a mean age of $59 \pm 7.2$ years. No significant differences were found between the asymptomatic, 

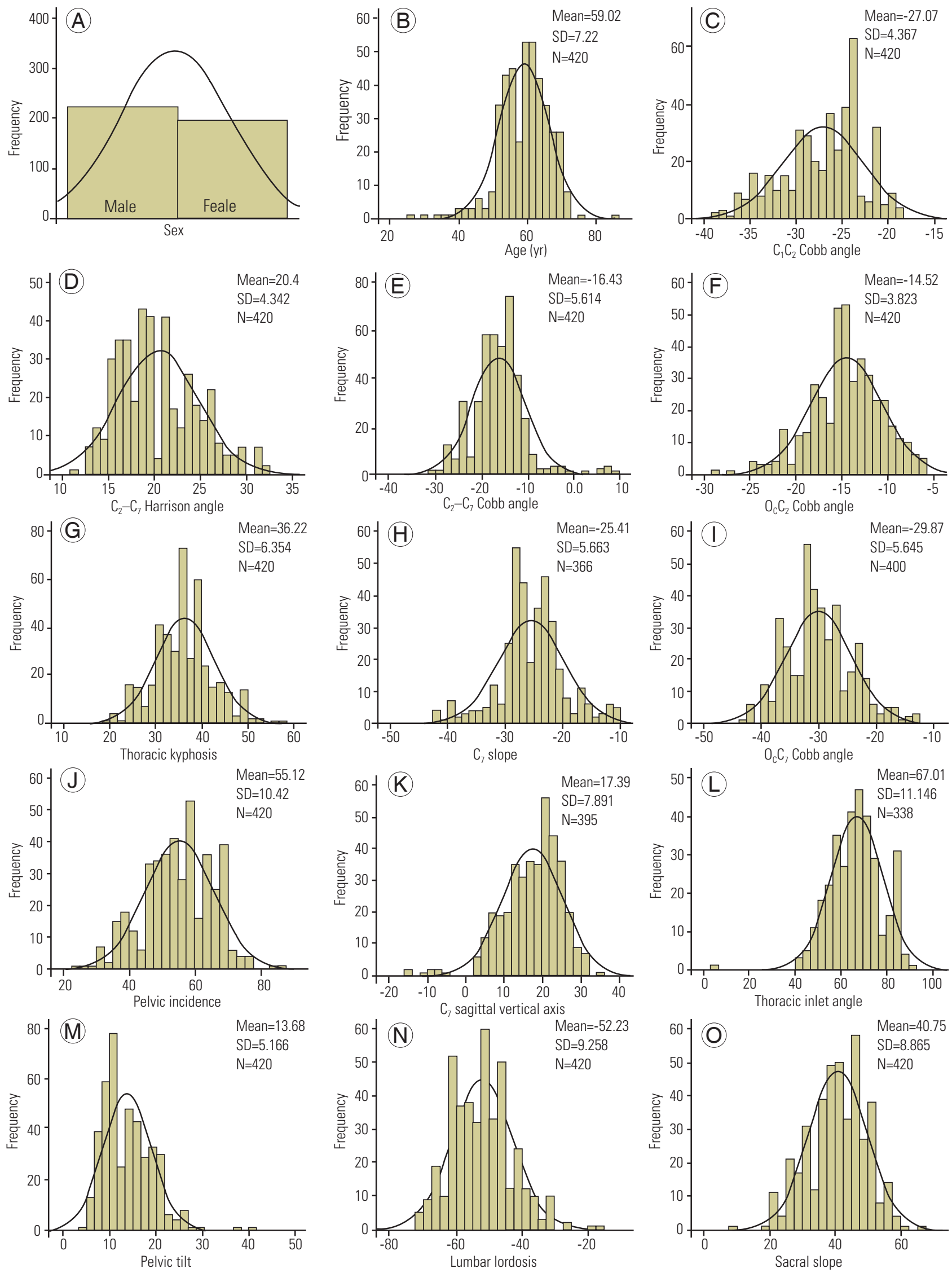

Fig. 1. (A-0) Histogram of normality. SD, standard deviation. 
Table 3. Radiographic measurements

\begin{tabular}{|c|c|c|c|c|c|}
\hline Variable & Asymptomatic & Lumbar group & Cervical group & Post-surgical group & $p$-value \\
\hline $\mathrm{C}_{1} \mathrm{C}_{2}$ Cobb angle & $-26.7 \pm 4.3$ & $-26.3 \pm 4.07$ & $-27.3 \pm 4.5$ & $-27.8 \pm 4.4$ & 0.053 \\
\hline $\mathrm{O}_{c} \mathrm{C}_{2}$ Cobb angle & $-14.7 \pm 3.5$ & $-14.3 \pm 3.7$ & $-14.4 \pm 4$ & $-14.5 \pm 3.8$ & 0.9 \\
\hline $\mathrm{O}_{\mathrm{c}} \mathrm{C}_{7}$ Cobb angle & $-29.3 \pm 6.9$ & $-30.2 \pm 4.9$ & $-29.5 \pm 5.3$ & $-30.2 \pm 5.2$ & 0.5 \\
\hline $\mathrm{C}_{2} \mathrm{C}_{7}$ Harrison angle & $20.1 \pm 4.1$ & $20.6 \pm 4.1$ & $21.1 \pm 4.7$ & $19.7 \pm 4.1$ & 0.1 \\
\hline $\mathrm{C}_{2} \mathrm{C}_{7}$ Cobb angle & $-15.4 \pm 6.7$ & $-16.8 \pm 5$ & $-17.1 \pm 5.5$ & $-16.34 \pm 4.9$ & 0.1 \\
\hline C7 slope angle & $-26.03 \pm 5.5$ & $-25.8 \pm 5.4$ & $-24.8 \pm 6.1$ & $-24.8 \pm 5.4$ & 0.3 \\
\hline $\mathrm{C}_{7}$ sagittal vertical axis $(\mathrm{mm})$ & $16.5 \pm 8.7$ & $17.3 \pm 8$ & $18.3 \pm 7.9$ & $17.3 \pm 6.7$ & 0.4 \\
\hline Thoracic kyphosis & $35.2 \pm 5.7$ & $36.9 \pm 7.4$ & $37.1 \pm 6$ & $35.6 \pm 5.9$ & 0.06 \\
\hline Thoracic inlet angle & $68.4 \pm 12$ & $66.1 \pm 10.2$ & $68.1 \pm 10.4$ & $65 \pm 11.8$ & 0.15 \\
\hline Pelvic incidence & $53.5 \pm 10.4$ & $56 \pm 9.2$ & $55.1 \pm 11.5$ & $55.6 \pm 10.2$ & 0.3 \\
\hline Sacral slope & $39.1 \pm 8.3$ & $41 \pm 8.7$ & $40.3 \pm 9.5$ & $42.4 \pm 8.5$ & 0.054 \\
\hline Pelvic tilt & $13.9 \pm 5.1$ & $13.9 \pm 5.3$ & $13.9 \pm 5.7$ & $12.8 \pm 4.2$ & 0.3 \\
\hline Lumbar lordosis & $-53.7 \pm 8.7$ & $-52.5 \pm 9.2$ & $-51.7 \pm 10.1$ & $-50.8 \pm 8.9$ & 0.1 \\
\hline
\end{tabular}

Values are presented as mean \pm standard deviation.

Table 4. Correlations obtained among measured parameters in the cervical group (correlation coefficient)

\begin{tabular}{|c|c|c|c|c|c|c|c|c|c|c|c|c|c|c|}
\hline & Age & $\mathrm{O}_{\mathrm{c}} \mathrm{C}_{2}$ & $\mathrm{O}_{\mathrm{c}} \mathrm{C}_{7}$ & $\mathrm{C}_{2} \mathrm{C}_{7} \mathrm{H}$ & $\mathrm{C}_{1} \mathrm{C}_{2}$ & $\mathrm{C}_{7} \mathrm{~S}$ & $\mathrm{C}_{2} \mathrm{C}_{7 \mathrm{c}}$ & TK & TIA & $\mathrm{PI}$ & SS & $\mathrm{LL}$ & PT & $\mathrm{C}_{7} \mathrm{SVA}$ \\
\hline Age & 1 & 0.166 & 0.017 & $0.200^{*}$ & 0.067 & 0.053 & $-0.200^{*}$ & -0.076 & -0.089 & 0.043 & 0.007 & 0.026 & 0.071 & 0.194 \\
\hline $\mathrm{O}_{\mathrm{c}} \mathrm{C}_{2}$ & & 1 & -0.120 & 0.134 & 0.189 & -0.092 & -0.117 & -0.126 & 0.006 & -0.075 & -0.057 & 0.075 & 0.041 & $0.199 *$ \\
\hline $\mathrm{O}_{\mathrm{c}} \mathrm{C}_{7}$ & & & 1 & -0.026 & -0.099 & 0.128 & -0.097 & 0.161 & -0.020 & 0.096 & 0.072 & -0.038 & 0.078 & -0.020 \\
\hline $\mathrm{C}_{2} \mathrm{C}_{7} \mathrm{H}$ & & & & 1 & -0.188 & -0.160 & $-0.845^{* *}$ & 0.033 & 0.135 & 0.112 & 0.061 & -0.103 & 0.176 & $0.757^{* *}$ \\
\hline $\mathrm{C}_{1} \mathrm{C}_{2}$ & & & & & 1 & -0.191 & 0.185 & $-0.238^{*}$ & -0.052 & $-0.226^{*}$ & -0.171 & $0.297^{* *}$ & $-0.215^{*}$ & -0.090 \\
\hline $\mathrm{C}_{7} \mathrm{~S}$ & & & & & & 1 & 0.084 & $0.218^{*}$ & -0.147 & 0.145 & $0.212^{*}$ & -0.171 & -0.050 & -0.189 \\
\hline $\mathrm{C}_{2} \mathrm{C}_{7 \mathrm{c}}$ & & & & & & & 1 & 0.027 & -0.117 & -0.100 & -0.033 & 0.097 & $-0.215^{*}$ & $-0.814^{* *}$ \\
\hline TK & & & & & & & & 1 & -0.082 & 0.098 & 0.114 & -0.114 & 0.092 & 0.111 \\
\hline TIA & & & & & & & & & 1 & 0.066 & -0.014 & 0.036 & 0.149 & 0.101 \\
\hline $\mathrm{Pl}$ & & & & & & & & & & 1 & $0.858^{* *}$ & $-0.866^{* *}$ & $0.488^{* *}$ & 0.083 \\
\hline SS & & & & & & & & & & & 1 & $-0.764^{* *}$ & 0.058 & 0.047 \\
\hline $\mathrm{LL}$ & & & & & & & & & & & & 1 & $-0.412^{* *}$ & -0.050 \\
\hline PT & & & & & & & & & & & & & 1 & 0.163 \\
\hline C7SVA & & & & & & & & & & & & & & 1 \\
\hline
\end{tabular}

$\mathrm{O}_{C} \mathrm{C}_{2 \mathrm{C}}$, occiput- $\mathrm{C}_{2}$ Cobb angle; $\mathrm{C}_{2} \mathrm{C}_{7} \mathrm{H}, \mathrm{C}_{2}-\mathrm{C}_{7}$ Harrison angle; $\mathrm{C}_{1} \mathrm{C}_{2 \mathrm{C}}, \mathrm{C}_{1}-\mathrm{C}_{2}$ Cobb angle; $\mathrm{C}_{7} \mathrm{~S}, \mathrm{C}_{7}$ slope; $\mathrm{C}_{2} \mathrm{C}_{7 \mathrm{C}}, \mathrm{C}_{2}-\mathrm{C}_{7}$ Cobb angle; TK, thoracic kyphosis; $\mathrm{TIA}$, thoracic inlet angle; PI, pelvic incidence; SS, sacral slope; LL, lumbar lordosis; PT, pelvic tilt; $\mathrm{C}_{7} \mathrm{SVA}$, C7 sagittal vertical axis.

$p<0.05 .{ }^{* *} p<0.01$.

lumbar, cervical, and post-surgical groups, in terms of age and gender. Demographic parameters are given in Table 2.

\section{Descriptive results of the spinopelvic parameters}

The means of PI, SS, PT, and LL were $55.1^{\circ} \pm 10.4^{\circ}$, $40.7^{\circ} \pm 8.8^{\circ}, 13.6^{\circ} \pm 5.1^{\circ}$, and $-52.2^{\circ} \pm 9.2^{\circ}$, respectively. Table
3 summarizes the diagnostic group-stratified means and standard deviations of the performed measurements.

\section{Descriptive results of the thoracic parameters}

The mean TIA was $67^{\circ} \pm 10.6^{\circ}$ (range, $41.5^{\circ}-91.5^{\circ}$ ), and the mean TK was $36.2^{\circ} \pm 6.3^{\circ}$ (range, $19.5^{\circ}-56.8^{\circ}$ ) (Table 3 ). 
Table 5. Correlations obtained among measured parameters in the post-surgical group (correlation coefficient)

\begin{tabular}{|c|c|c|c|c|c|c|c|c|c|c|c|c|c|c|}
\hline & Age & $\mathrm{O}_{\mathrm{c}} \mathrm{C}_{2}$ & $\mathrm{O}_{\mathrm{c}} \mathrm{C}_{7}$ & $\mathrm{C}_{2} \mathrm{C}_{7} \mathrm{H}$ & $\mathrm{C}_{1} \mathrm{C}_{2}$ & $\mathrm{C}_{7} \mathrm{~S}$ & $\mathrm{C}_{2} \mathrm{C}_{7 \mathrm{c}}$ & TK & TIA & $\mathrm{PI}$ & SS & LL & $\mathrm{PT}$ & $\mathrm{C}_{7} \mathrm{SVA}$ \\
\hline Age & 1 & -0.146 & -0.028 & -0.109 & -0.176 & -0.024 & 0.015 & -0.007 & 0.046 & $-0.193^{*}$ & -0.150 & 0.042 & -0.08 & 0.019 \\
\hline $\mathrm{O}_{\mathrm{c}} \mathrm{C}_{2}$ & & 1 & $0.213^{*}$ & 0.072 & $0.234^{*}$ & 0.028 & -0.010 & -0.043 & 0.049 & -0.186 & -0.143 & 0.096 & -0.087 & 0.037 \\
\hline $\mathrm{O}_{\mathrm{c}} \mathrm{C}_{7}$ & & & 1 & 0.031 & $0.209^{*}$ & 0.155 & -0.067 & 0.078 & 0.157 & $0.229^{*}$ & $0.258^{*}$ & -0.144 & 0.000 & -0.111 \\
\hline $\mathrm{C}_{2} \mathrm{C}_{7} \mathrm{H}$ & & & & 1 & -0.051 & 0.098 & $-0.737^{* *}$ & $0.234^{*}$ & 0.125 & $0.232^{*}$ & $0.214^{*}$ & -0.142 & 0.05 & $0.725^{* *}$ \\
\hline $\mathrm{C}_{1} \mathrm{C}_{2}$ & & & & & 1 & -0.074 & 0.156 & -0.017 & 0.072 & 0.027 & 0.072 & 0.090 & $-0.212^{*}$ & -0.178 \\
\hline $\mathrm{C}_{7} \mathrm{~S}$ & & & & & & 1 & -0.134 & 0.088 & -0.091 & 0.155 & 0.143 & -0.139 & 0.061 & 0.118 \\
\hline $\mathrm{C}_{2} \mathrm{C}_{7 \mathrm{c}}$ & & & & & & & 1 & -0.151 & -0.085 & -0.159 & -0.159 & 0.152 & 0.059 & $-0.605^{* *}$ \\
\hline TK & & & & & & & & 1 & $-0.371 * *$ & 0.019 & 0.036 & -0.084 & -0.074 & 0.125 \\
\hline TIA & & & & & & & & & 1 & -0.114 & -0.125 & 0.140 & 0.009 & 0.153 \\
\hline $\mathrm{Pl}$ & & & & & & & & & & 1 & $0.922^{* *}$ & $-0.793^{* *}$ & $0.345^{* *}$ & 0.111 \\
\hline SS & & & & & & & & & & & 1 & $-0.713^{* *}$ & 0.145 & 0.082 \\
\hline $\mathrm{LL}$ & & & & & & & & & & & & 1 & $-0.293^{* *}$ & -0.050 \\
\hline PT & & & & & & & & & & & & & 1 & 0.168 \\
\hline $\mathrm{C}_{7}$ SVA & & & & & & & & & & & & & & 1 \\
\hline
\end{tabular}

$\mathrm{O}_{\mathrm{C}} \mathrm{C}_{2 \mathrm{C}}$, occiput- $\mathrm{C}_{2}$ Cobb angle; $\mathrm{C}_{2} \mathrm{C}_{7} \mathrm{H}, \mathrm{C}_{2}-\mathrm{C}_{7}$ Harrison angle; $\mathrm{C}_{1} \mathrm{C}_{2 \mathrm{C}}, \mathrm{C}_{1}-\mathrm{C}_{2}$ Cobb angle; $\mathrm{C}_{7} \mathrm{~S}, \mathrm{C}_{7}$ slope; $\mathrm{C}_{2} \mathrm{C}_{7 \mathrm{C}}, \mathrm{C}_{2}-\mathrm{C}_{7}$ Cobb angle; TK, thoracic kyphosis; TIA, thoracic inlet angle; PI, pelvic incidence; SS, sacral slope; LL, lumbar lordosis; PT, pelvic tilt; $C_{7} S V A, C 7$ sagittal vertical axis.

$p<0.05 .{ }^{* *} p<0.01$.

Table 6. Correlations obtained among measured parameters in the asymptomatic group (correlation coefficient)

\begin{tabular}{|c|c|c|c|c|c|c|c|c|c|c|c|c|c|c|}
\hline & Age & $\mathrm{O}_{\mathrm{c}} \mathrm{C}_{2}$ & $\mathrm{O}_{\mathrm{c}} \mathrm{C}_{7}$ & $\mathrm{C}_{2} \mathrm{C}_{7} \mathrm{H}$ & $\mathrm{C}_{1} \mathrm{C}_{2}$ & $\mathrm{C}_{7} \mathrm{~S}$ & $\mathrm{C}_{2} \mathrm{C}_{7 \mathrm{c}}$ & TK & TIA & $\mathrm{PI}$ & SS & $\mathrm{LL}$ & $\mathrm{PT}$ & $\mathrm{C}_{7} \mathrm{SVA}$ \\
\hline Age & 1 & 0.132 & -0.055 & 0.124 & -0.006 & -0.124 & -0.096 & 0.011 & -0.074 & 0.039 & 0.123 & 0.013 & -0.113 & 0.097 \\
\hline $\mathrm{O}_{\mathrm{c}} \mathrm{C}_{2}$ & & 1 & -0.032 & 0.150 & $0.231^{*}$ & $-0.283^{* *}$ & -0.061 & -0.018 & -0.174 & -0.152 & -0.183 & $0.256^{* *}$ & 0.071 & 0.114 \\
\hline $\mathrm{O}_{\mathrm{c}} \mathrm{C}_{7}$ & & & 1 & $-0.211^{*}$ & 0.173 & -0.069 & 0.047 & 0.145 & 0.088 & 0.021 & 0.015 & -0.088 & -0.149 & 0.016 \\
\hline $\mathrm{C}_{2} \mathrm{C}_{7} \mathrm{H}$ & & & & 1 & 0.135 & $-0.247^{*}$ & $-0.432^{* *}$ & 0.080 & -0.099 & 0.065 & 0.030 & -0.043 & 0.169 & $0.495^{* *}$ \\
\hline $\mathrm{C}_{1} \mathrm{C}_{2}$ & & & & & 1 & -0.092 & -0.038 & 0.102 & -0.09 & -0.097 & -0.120 & 0.114 & -0.099 & 0.142 \\
\hline $\mathrm{C}_{7} \mathrm{~S}$ & & & & & & 1 & $0.223^{*}$ & 0.02 & 0.122 & -0.054 & -0.016 & 0.026 & -0.032 & -0.123 \\
\hline $\mathrm{C}_{2} \mathrm{C}_{7 \mathrm{c}}$ & & & & & & & 1 & -0.092 & 0.055 & 0.105 & 0.103 & -0.127 & 0.020 & $-0.685^{* *}$ \\
\hline TK & & & & & & & & 1 & 0.094 & 0.041 & -0.040 & 0.025 & 0.054 & 0.183 \\
\hline TIA & & & & & & & & & 1 & -0.042 & -0.085 & 0.045 & 0.090 & 0.000 \\
\hline $\mathrm{PI}$ & & & & & & & & & & 1 & $0.896^{* *}$ & $-0.778^{* *}$ & $0.468^{* *}$ & -0.079 \\
\hline SS & & & & & & & & & & & 1 & $-0.741^{* *}$ & 0.163 & -0.036 \\
\hline $\mathrm{LL}$ & & & & & & & & & & & & 1 & $-0.291^{* *}$ & 0.064 \\
\hline PT & & & & & & & & & & & & & 1 & -0.098 \\
\hline $\mathrm{C}_{7} \mathrm{SVA}$ & & & & & & & & & & & & & & 1 \\
\hline
\end{tabular}

$\mathrm{O}_{c} \mathrm{C}_{2 \mathrm{C}}$, occiput- $\mathrm{C}_{2}$ Cobb angle; $\mathrm{C}_{2} \mathrm{C}_{7} \mathrm{H}, \mathrm{C}_{2}-\mathrm{C}_{7}$ Harrison angle; $\mathrm{C}_{1} \mathrm{C}_{2 \mathrm{C}}, \mathrm{C}_{1}-\mathrm{C}_{2}$ Cobb angle; $\mathrm{C}_{7} \mathrm{~S}, \mathrm{C}_{7}$ slope; $\mathrm{C}_{2} \mathrm{C}_{7 \mathrm{C}}, \mathrm{C}_{2}-\mathrm{C}_{7}$ Cobb angle; TK, thoracic kyphosis; TIA, thoracic inlet angle; PI, pelvic incidence; SS, sacral slope; LL, lumbar lordosis; PT, pelvic tilt; $C_{7} S V A$, C7 sagittal vertical axis.

${ }^{*} p<0.05 .{ }^{* *} p<0.01$.

\section{Descriptive results of the cervical and occipital parameters}

The average $\mathrm{C}_{1} \mathrm{C}_{2}$ Cobb angle was $-27.07^{\circ} \pm 4.3^{\circ}, \mathrm{O}_{\mathrm{C}} \mathrm{C}_{2}$ Cobb angle was $-14.5^{\circ} \pm 3.8^{\circ}, \mathrm{O}_{\mathrm{C}} \mathrm{C}_{7} \mathrm{Cobb}$ angle was $-29.8^{\circ} \pm 5.6^{\circ}, \mathrm{C}_{2} \mathrm{C}_{7}$ Harrison angle was $20.4^{\circ} \pm 4.3^{\circ}, \mathrm{C}_{2} \mathrm{C}_{7}$ Cobb angle was $-16.4^{\circ} \pm 5.6^{\circ}$, and $C_{7}$ slope was $-25.4^{\circ} \pm 5.6^{\circ}$. The analysis of these parameters revealed no statistically significant difference between the four groups (Table 3). 
Table 7. Correlations obtained among measured parameters in lumber group (correlation coefficient)

\begin{tabular}{|c|c|c|c|c|c|c|c|c|c|c|c|c|c|c|}
\hline & Age & $\mathrm{O}_{\mathrm{c}} \mathrm{C}_{2}$ & $\mathrm{O}_{\mathrm{c}} \mathrm{C}_{7}$ & $\mathrm{C}_{2} \mathrm{C}_{7} \mathrm{H}$ & $\mathrm{C}_{1} \mathrm{C}_{2}$ & $\mathrm{C}_{7} \mathrm{~S}$ & $\mathrm{C}_{2} \mathrm{C}_{7 \mathrm{c}}$ & TK & TIA & $\mathrm{PI}$ & SS & LL & PT & $\mathrm{C}_{7} \mathrm{SVA}$ \\
\hline Age & 1 & -0.102 & -0.037 & 0.130 & 0.053 & -0.131 & -0.073 & -0.088 & -0.027 & -0.135 & $-0.247^{*}$ & 0.110 & 0.025 & 0.094 \\
\hline $\mathrm{O}_{\mathrm{c}} \mathrm{C}_{2}$ & & 1 & -0.043 & 0.179 & 0.035 & 0.005 & -0.158 & 0.082 & 0.131 & $-0.200^{*}$ & $-0.215^{*}$ & 0.146 & -0.057 & 0.059 \\
\hline $\mathrm{O}_{\mathrm{c}} \mathrm{C}_{7}$ & & & 1 & 0.115 & 0.151 & -0.072 & 0.003 & 0.064 & 0.049 & 0.143 & 0.006 & -0.162 & 0.111 & 0.054 \\
\hline $\mathrm{C}_{2} \mathrm{C}_{7} \mathrm{H}$ & & & & 1 & -0.074 & 0.021 & $-0.758^{* *}$ & -0.013 & 0.164 & -0.023 & -0.171 & 0.088 & 0.133 & $0.689^{* *}$ \\
\hline $\mathrm{C}_{1} \mathrm{C}_{2}$ & & & & & 1 & 0.064 & 0.083 & 0.158 & -0.036 & 0.026 & -0.090 & -0.017 & 0.115 & -0.124 \\
\hline $\mathrm{C}_{7} \mathrm{~S}$ & & & & & & 1 & 0.014 & $0.250^{*}$ & 0.102 & 0.057 & 0.053 & -0.005 & -0.023 & -0.030 \\
\hline $\mathrm{C}_{2} \mathrm{C}_{7 \mathrm{c}}$ & & & & & & & 1 & -0.027 & -0.162 & -0.012 & 0.100 & -0.035 & -0.165 & $-0.804^{* *}$ \\
\hline TK & & & & & & & & 1 & -0.047 & -0.098 & $-0.223^{*}$ & 0.033 & 0.160 & -0.049 \\
\hline TIA & & & & & & & & & 1 & 0.199 & 0.096 & -0.135 & 0.136 & 0.019 \\
\hline $\mathrm{PI}$ & & & & & & & & & & 1 & $0.797^{* *}$ & $-0.576^{* *}$ & $0.267^{* *}$ & -0.022 \\
\hline SS & & & & & & & & & & & 1 & $-0.449^{* *}$ & -0.145 & -0.073 \\
\hline $\mathrm{LL}$ & & & & & & & & & & & & 1 & -0.182 & 0.040 \\
\hline PT & & & & & & & & & & & & & 1 & 0.102 \\
\hline $\mathrm{C}_{7}$ SVA & & & & & & & & & & & & & & 1 \\
\hline
\end{tabular}

$\mathrm{O}_{\mathrm{C}} \mathrm{C}_{2 \mathrm{C}}$, occiput- $\mathrm{C}_{2}$ Cobb angle; $\mathrm{C}_{2} \mathrm{C}_{7} \mathrm{H}, \mathrm{C}_{2}-\mathrm{C}_{7}$ Harrison angle; $\mathrm{C}_{1} \mathrm{C}_{2 \mathrm{C}}, \mathrm{C}_{1}-\mathrm{C}_{2}$ Cobb angle; $\mathrm{C}_{7} \mathrm{~S}, \mathrm{C}_{7}$ slope; $\mathrm{C}_{2} \mathrm{C}_{7 \mathrm{C}}, \mathrm{C}_{2}-\mathrm{C}_{7}$ Cobb angle; TK, thoracic kyphosis; TIA, thoracic inlet angle; PI, pelvic incidence; SS, sacral slope; LL, lumbar lordosis; PT, pelvic tilt; $C_{7} S V A$, C7 sagittal vertical axis. " $p<0.05 .{ }^{* *} p<0.01$.

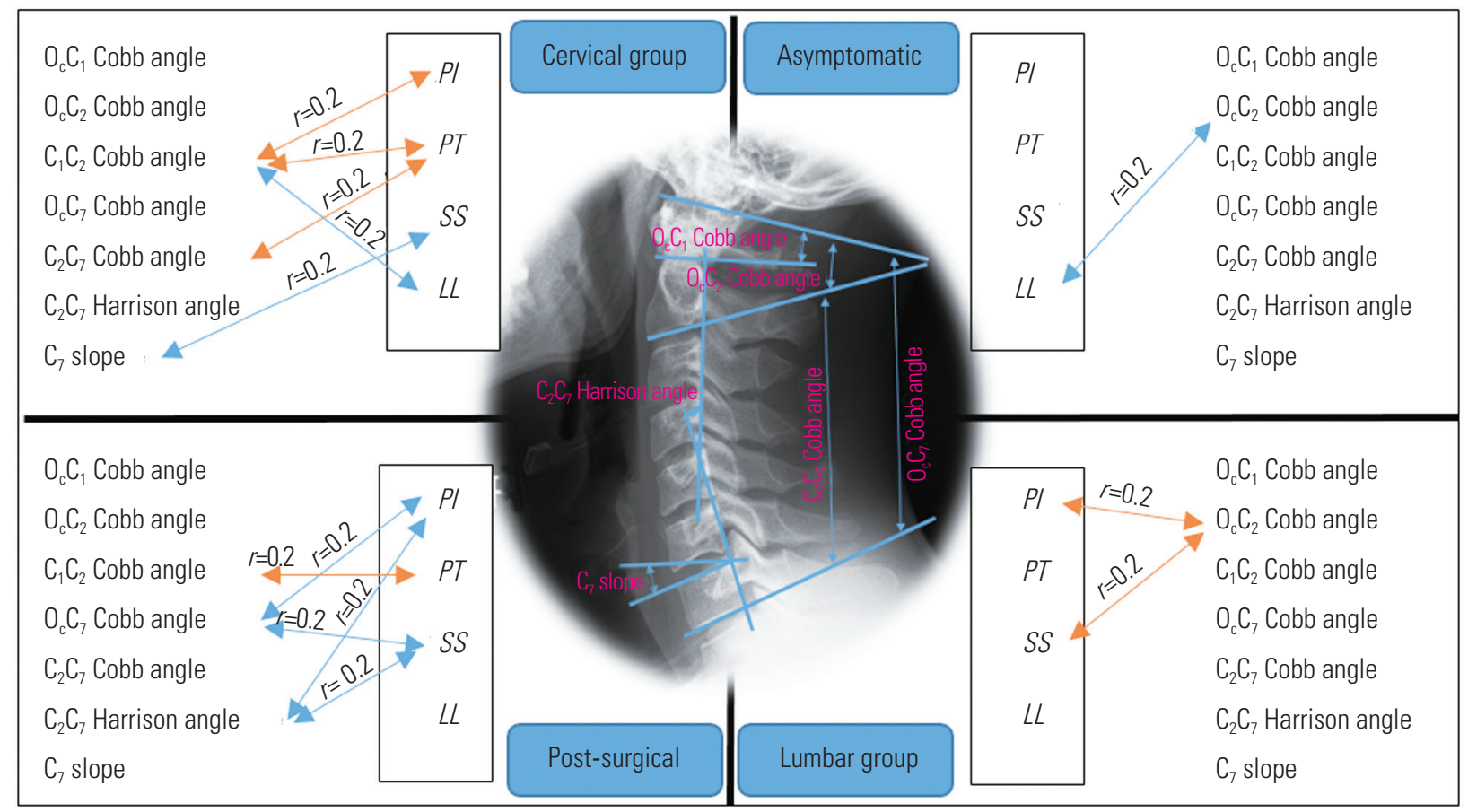

Fig. 2. Schematic drawing of the correlations between spinopelvic and cervical parameter. $\mathrm{O}_{\mathrm{C}} \mathrm{C}_{2 \mathrm{C}}$, occiput- $\mathrm{C}_{2}$ Cobb angle; $\mathrm{C}_{2} \mathrm{C}_{7} \mathrm{H}, \mathrm{C}_{2}-\mathrm{C}_{7} \mathrm{Harrison}$ angle; $C_{1} C_{2 c}, C_{1}-C_{2}$ Cobb angle; $C_{7} S, C_{7}$ slope; $C_{2} C_{7 C}, C_{2}-C_{7}$ Cobb angle; Pl, pelvic incidence; $S S$, sacral slope; LL, lumbar lordosis; $P$ T, pelvic tilt.

\section{The relationship between the age and cervical spine parameters}

In terms of the cervical parameters, only $\mathrm{C}_{2} \mathrm{C}_{7}$ Cobb angle correlated with age in the cervical group (Table 4). In the other studied groups, there was no correlation between age and cervical parameters. 
Table 8. Correlations obtained among measured parameters for all patient (correlation coefficient)

\begin{tabular}{|c|c|c|c|c|c|c|c|c|c|c|c|c|c|c|}
\hline & Age & $\mathrm{O}_{\mathrm{c}} \mathrm{C}_{2}$ & $\mathrm{O}_{\mathrm{c}} \mathrm{C}_{7}$ & $\mathrm{C}_{2} \mathrm{C}_{7} \mathrm{H}$ & $\mathrm{C}_{1} \mathrm{C}_{2}$ & $\mathrm{C}_{7} \mathrm{~S}$ & $\mathrm{C}_{2} \mathrm{C}_{7 \mathrm{c}}$ & TK & TIA & PI & SS & LL & PT & $\mathrm{C}_{7} \mathrm{SVA}$ \\
\hline Age & 1 & 0.025 & -0.033 & 0.091 & -0.006 & -0.053 & $-0.096^{*}$ & -0.046 & -0.055 & -0.039 & -0.047 & 0.049 & -0.020 & $0.104^{*}$ \\
\hline $\mathrm{O}_{\mathrm{c}} \mathrm{C}_{2}$ & & 1 & 0.001 & $0.135^{* *}$ & $0.173^{* *}$ & -0.083 & -0.088 & -0.018 & 0.001 & $-0.145^{* *}$ & $-0.141^{* *}$ & $0.137^{* *}$ & -0.004 & $0.107^{*}$ \\
\hline $\mathrm{O}_{\mathrm{c}} \mathrm{C}_{7}$ & & & 1 & -0.033 & $0.109^{*}$ & 0.027 & -0.014 & $0.106^{*}$ & 0.078 & $0.106^{*}$ & 0.073 & $-0.107^{*}$ & 0.006 & -0.009 \\
\hline $\mathrm{C}_{2} \mathrm{C}_{7} \mathrm{H}$ & & & & 1 & -0.044 & -0.086 & $-0.674^{* *}$ & 0.087 & 0.083 & $0.100^{*}$ & 0.027 & -0.053 & $0.144^{* *}$ & $0.660^{* *}$ \\
\hline $\mathrm{C}_{1} \mathrm{C}_{2}$ & & & & & 1 & -0.092 & 0.089 & 0.008 & -0.031 & -0.078 & -0.089 & $0.115^{*}$ & -0.092 & -0.055 \\
\hline $\mathrm{C}_{7} \mathrm{~S}$ & & & & & & 1 & 0.062 & $0.154^{* *}$ & -0.006 & 0.078 & $0.110^{*}$ & -0.066 & -0.026 & -0.070 \\
\hline $\mathrm{C}_{2} \mathrm{C}_{7 \mathrm{c}}$ & & & & & & & 1 & -0.071 & -0.059 & -0.040 & 0.002 & 0.007 & -0.08 & $-0.728^{* *}$ \\
\hline TK & & & & & & & & 1 & -0.085 & 0.021 & -0.034 & -0.030 & 0.075 & 0.086 \\
\hline TIA & & & & & & & & & 1 & 0.025 & -0.035 & 0.005 & $0.100^{*}$ & 0.054 \\
\hline $\mathrm{Pl}$ & & & & & & & & & & 1 & $0.866^{* *}$ & $-0.745^{* *}$ & $0.393^{* *}$ & 0.024 \\
\hline SS & & & & & & & & & & & 1 & $-0.643^{* *}$ & 0.036 & 0.006 \\
\hline $\mathrm{LL}$ & & & & & & & & & & & & 1 & $-0.304^{* *}$ & 0.008 \\
\hline PT & & & & & & & & & & & & & 1 & 0.076 \\
\hline $\mathrm{C}_{7}$ SVA & & & & & & & & & & & & & & 1 \\
\hline
\end{tabular}

$\mathrm{O}_{\mathrm{C}} \mathrm{C}_{2 \mathrm{C}}$, occiput- $\mathrm{C}_{2}$ Cobb angle; $\mathrm{C}_{2} \mathrm{C}_{7} \mathrm{H}, \mathrm{C}_{2}-\mathrm{C}_{7}$ Harrison angle; $\mathrm{C}_{1} \mathrm{C}_{2 \mathrm{C}}, \mathrm{C}_{1}-\mathrm{C}_{2}$ Cobb angle; $\mathrm{C}_{7} \mathrm{~S}, \mathrm{C}_{7}$ slope; $\mathrm{C}_{2} \mathrm{C}_{7 \mathrm{C}}, \mathrm{C}_{2}-\mathrm{C}_{7}$ Cobb angle; TK, thoracic kyphosis; TIA, thoracic inlet angle; PI, pelvic incidence; SS, sacral slope; LL, lumbar lordosis; PT, pelvic tilt; $C_{7} S V A, C 7$ sagittal vertical axis. " $p<0.05 .{ }^{* *} p<0.01$.

\section{The relationship between the spinopelvic and cervical spine parameters}

The subjects in the cervical group showed positive correlation between the $\mathrm{C}_{7}$ slope and SS $(r=0.212, p=0.039)$ and a negative correlation between $\mathrm{C}_{2} \mathrm{C}_{7}$ Cobb angle and PT $(r=-0.2, p=0.02)$. Also, the cervical group showed a statistically significant correlation between the $\mathrm{C}_{1} \mathrm{C}_{2}$ Cobb angle and three spinopelvic parameters (LL: $r=0.2$, $p=0.002$; PI: $r=-0.2, p=0.02$; and PT: $r=-0.2, p=0.02$ ) (Table 4 ). In the post-surgical group, PI and SS were two spinopelvic parameters that showed a significant positive correlation to $\mathrm{O}_{\mathrm{C}} \mathrm{C}_{7}$ Cobb angle and $\mathrm{C}_{2} \mathrm{C}_{7}$ Harrison angle $(r=0.2)$ (Table 5). Moreover, $\mathrm{C}_{1} \mathrm{C}_{2}$ Cobb angle correlated to PT in the post-surgical group $(r=-0.2, p=0.01)$. Asymptomatic subjects and the lumbar group did not show any significant correlation between the spinopelvic and cervical parameters (Tables 6, 7, Fig. 2).

Regardless of the subgroups $(n=420)$, there was a statistically significant correlation between the $\mathrm{C}_{2} \mathrm{C}_{7}$ Harrison angle and $\mathrm{PT}, \mathrm{C}_{2} \mathrm{C}_{7}$ Harrison angle and PI, $\mathrm{C}_{1} \mathrm{C}_{2}$ Cobb angle and $\mathrm{LL}$, and $\mathrm{C}_{7}$ slope and SS. However, there was no correlation between the $\mathrm{C}_{2} \mathrm{C}_{7}$ Cobb angle and spinopelvic parameters (Table 8).

\section{The relationship between the thoracic and cervical spine parameters}

There was significant correlation between $\mathrm{C}_{7}$ slope and TK in the lumbar $(r=0.2, p=0.01)$ and cervical $(r=0.2, p=0.03)$ groups. The TK correlated significantly with the $\mathrm{C}_{1} \mathrm{C}_{2} \mathrm{Cobb}$ angle in the cervical group $(r=0.2, p=0.01)$ and with the $\mathrm{C}_{2} \mathrm{C}_{7}$ Harrison angle in the post-surgical subjects (Tables 4, $5,7)$. As with the spinopelvic parameters, there was no correlation between the thoracic and cervical spine parameters in the asymptomatic group (Table 6). Regardless of the subgroups, $\mathrm{C}_{7}$ slope was the only cervical parameter that was associated with TK in all the study subjects (Table 8).

\section{The relationship between the occipital and cervical spine parameters}

Statistically significant correlation was observed between the $\mathrm{O}_{\mathrm{C}} \mathrm{C}_{2}$ Cobb angle and $\mathrm{C}_{1} \mathrm{C}_{2}$ Cobb angle $(r=0.2, p=0.01)$ and between the $\mathrm{O}_{\mathrm{C}} \mathrm{C}_{2}$ Cobb angle and $\mathrm{C}_{7}$ slope $(r=0.283$, $p=0.006$ ) in the asymptomatic subjects (Table 6 ). The lumbar and cervical groups did not show any significant correlation between the occipital and cervical parameters (Tables 4, 7). Regardless of the subgroups, both studied occipital parameters correlated with the $\mathrm{C}_{1} \mathrm{C}_{2}$ Cobb angle 
$\left(\mathrm{O}_{\mathrm{C}} \mathrm{C}_{2}\right.$ Cobb angle: $r=0.173, p=0.000$ and $\mathrm{O}_{\mathrm{C}} \mathrm{C}_{7} \mathrm{Cobb}$ angle: $r=0.1, p=0.02$ ) (Table 8 ).

\section{Multiple regression analysis and the linear regres- sion model}

Multiple linear regression analysis was used regarding $\mathrm{C}_{0} \mathrm{C}_{7}$ angle $(\mathrm{CL})$ as a dependent variable and regarding $\mathrm{C}_{0} \mathrm{C}_{2}, \mathrm{TK}, \mathrm{PI}$, and LL as independent variables to model the relationship between $\mathrm{C}_{0} \mathrm{C}_{7}$ angle and potential factors by fitting a linear equation to the data. The equation for predicting the alignment of $\mathrm{CL}$ was as follows: $\mathrm{C}_{0} \mathrm{C}_{7}$ angle $=0.15\left(\mathrm{C}_{1} \mathrm{C}_{2}\right)+0.8 \mathrm{TK}+0.03 \mathrm{PI}-0.04 \mathrm{LL}-32$.

\section{Discussion}

In the past decades, much emphasis has been placed on identifying the sagittal profile of the thoracolumbar spine. In contrast to numerous discussions about the relationship between spinopelvic parameters and global sagittal balance of the spine, the correlation between spinopelvic parameters and cervical sagittal balance is unclear. The cervical spine is a relatively complex segment, and many factors influence its alignment and balance. The identification of compensatory mechanisms can be a precious point for the appropriate reconstruction of cervical spine alignment, especially in patients who have undergone spinal surgeries.

In terms of normal values, the measured values vary widely in different studies. It seems that factors, such as age, sex, race, and method of measuring parameters, cause this disparity. Nunez-Pereira et al. [14] studied the interdependences between occipitocervical and spinopelvic parameters. They reported mean values for $\mathrm{O}_{\mathrm{C}} \mathrm{C}_{2}$ and $\mathrm{C}_{2} \mathrm{C}_{7}$ Cobb angles as $-12.7^{\circ}$ and $-15.8^{\circ}$ for asymptomatic subjects, $-10.4^{\circ}$ and $18.9^{\circ}$ for patients with cervicalrelated symptoms, and $-11.9^{\circ}$ and $-21.2^{\circ}$ for post-surgical patients, respectively. In our study, $\mathrm{O}_{\mathrm{C}} \mathrm{C}_{2}$ angle was $-14.7^{\circ}$ for the asymptomatic patients, $-14.3^{\circ}$ for the patients with lumbar-related symptoms, $-14.4^{\circ}$ for those with cervicalrelated symptoms, and $-14.5^{\circ}$ for post-surgical patients. The $\mathrm{C}_{2} \mathrm{C}_{7}$ Cobb angle was $-15.4^{\circ},-16.8^{\circ},-17.1^{\circ}$, and $-16.34^{\circ}$ in our four study group, respectively. These results are similar to those of Nunez-Pereira et al. [14] relatively. Also, the total amount of $\mathrm{CL}\left(\mathrm{O}_{\mathrm{C}} \mathrm{C}_{7} \mathrm{Cobb}\right.$ angle $)$ in our study was similar to that of previous studies $\left(-29.8^{\circ} \pm 5.6^{\circ}\right)$ $[14,15]$. A study of Guo et al. [15] showed slight differ- ences with regard to age in the occipitocervical alignment of healthy volunteers. In our study, age correlation was observed only with $\mathrm{C}_{2} \mathrm{C}_{7}$ Cobb angle and $\mathrm{C}_{2} \mathrm{C}_{7}$ Harrison angle for patients with cervical-related symptoms. The age-related structural changes in the spine justify this difference.

Several studies have implied that the lumbar and pelvic parameters are correlated with the cervical region values. In our study, the mean value of LL was $-52.2^{\circ} \pm 9.2^{\circ}$, SS was $40.7^{\circ} \pm 8.8^{\circ}$, PI was $55.1^{\circ} \pm 10.4^{\circ}$, and PT was $13.6^{\circ} \pm 5.1^{\circ}$. The analysis showed no correlation between the spinopelvic and cervical parameters in asymptomatic and lumbar patients. However, $\mathrm{C}_{1} \mathrm{C}_{2} \mathrm{Cobb}$ angle correlated with the three spinopelvic parameters (LL: $r=0.2, p=0.002$; PI: $r=-0.2, p=0.02$; PT: $r=-0.2, p=0.02)$, and the $\mathrm{C}_{2} \mathrm{C}_{7} \mathrm{Cobb}$ angle correlated with PT $(r=0.2, p=0.02)$ in cervical symptomatic patients. These results are consistent with those of Nunez-Pereira et al. [14] and Lee et al. [16]. Shao et al. [17] performed measurements in asymptomatic healthy volunteers and found that CL correlated with $\operatorname{LL}(r=0.1, p<0.01)$ and $\mathrm{O}_{\mathrm{C}} \mathrm{C}_{2}$ Cobb angle correlated with SS $(r=-0.1, p<0.05)$. In our study, regardless of the patients' symptoms $(n=420)$, $\mathrm{C}_{1} \mathrm{C}_{2}$ Cobb angle correlated with LL $(r=0.1, p=0.01)$ and $\mathrm{C}_{2} \mathrm{C}_{7}$ Harrison angle correlated with PI $(r=0.1, p=0.04)$ and PT $(r=0.12, p=0.003)$. It seems that pain promotes compensatory flexion to increase spinal canal volume that leads to segmental alignment changes, and this can be a factor in the change of the correlation between spinal parameters in patients with cervical pathologies compared to the asymptomatic individual.

In our study, there was no significant correlation between $\mathrm{CL}\left(\mathrm{C}_{0} \mathrm{C}_{7} \mathrm{Cobb}\right.$ angle $)$ and spinopelvic parameters in asymptomatic, cervical symptomatic, and lumbar symptomatic patients. However, regardless of the patients' symptoms $(n=420)$, there was a significant relationship between $\mathrm{C}_{0} \mathrm{C}_{7}$ angle and PI and LL. This will clarify the key role of sample numbers in evaluating the correlation between the parameters.

Recently, Nunez-Pereira et al. [14] proposed $C_{7}$ slope as a predictor of the overall sagittal balance of the spine. They believe that if $\mathrm{C}_{7}$ slope is altered on cervical radiographs, a full-length sagittal radiograph should be taken to rule out the overall sagittal imbalance. However, we found a negative correlation between $\mathrm{C}_{7}$ slope and $\mathrm{C}_{7}$ SVA, and, in contrast to Nunez-Pereira et al. [14], this correlation was not statistically significant. Thus, we believe that further studies with higher sample sizes are needed to 
examine the role of $\mathrm{C}_{7}$ slope in the overall sagittal balance of the spine.

$\mathrm{C}_{7}$ SVA represents the global spinal sagittal alignment; thus, it should have a specific impact on CL to maintain sagittal balance, independent of other parameters. Subjects who had a positive SVA displayed an increase in CL, regardless of whether their SVA was within the normal range of values. This is a compensatory mechanism to maintain a horizontal gaze in response to changes in sagittal global alignment [11]. Interestingly, $\mathrm{C}_{7} \mathrm{SVA}$ was the only parameter in our study that showed a significant correlation with the $\mathrm{C}_{2} \mathrm{C}_{7}$ Cobb angle in the four groups ( $r=-0.6$ in asymptomatic; -0.8 in lumbar; -0.8 in cervical; and -0.6 in post-surgical subjects). However, there was no correlation between $\mathrm{C}_{7}$ SVA and total $\mathrm{CL}\left(\mathrm{O}_{\mathrm{C}} \mathrm{C}_{7} \mathrm{Cobb}\right.$ angle). Matsubayashi et al. [18] found a similar significant correlation $(r=0.5)$ in normal adults. They divided the $\mathrm{C}_{2} \mathrm{C}_{7}$ angle into the $\mathrm{C} 2-\mathrm{C} 4$ (middle cervical) and $\mathrm{C} 5-\mathrm{C} 7$ (lower cervical) angles and found that $\mathrm{C}_{7}$ SVA correlated with the $\mathrm{C} 5-\mathrm{C} 7$ angle but not with the $\mathrm{C} 2-\mathrm{C} 4$ angle.

The cervical spine is considered as a unique segment and can be divided into two sub-segments with different roles and functions. For example, the highest level of cervical flexion and rotation is seen in the upper cervical segment $\left(\mathrm{C}_{0} \mathrm{C}_{1}\right.$ and $\left.\mathrm{C}_{1} \mathrm{C}_{2}\right)$ whereas the lower segment $\left(\mathrm{C}_{2} \mathrm{C}_{7}\right)$ is associated with lateral bending $[19,20]$. Therefore, the difference in correlation between these two cervical subsegments and the spinopelvic parameters is not surprising. According to our study results, it seems that spinopelvic parameters (such as, PI, PT, and LL) show greater correlation with the upper cervical segment $\left(\mathrm{C}_{1} \mathrm{C}_{2} \mathrm{Cobb}\right.$ angle) and that the total $\mathrm{CL}$ and the sub-axial parameters (C3-C7) are less affected.

The prospective design and the survey of symptomatic subjects parallel to the asymptomatic individuals are the benefits of our study. This heterogeneity in patient selection facilitates the understanding of the general functions of the cervical spine, but it should be kept in mind that standard sampling and large sample size are the prerequisites of assessment of correlation in any population. Thus, the number of subjects may have underpowered our results. There are structural differences between population groups and races [19-21]. Therefore, this should be considered when comparing the results of various studies in different regions and races. We have not studied the correlation between some of the occipitocervical parameters, such as occipital incidence and occipital slope. It is therefore recommended to study their effect on cervical alignment in subsequent studies. Further study with a larger number of cases in various spinal disorders in different races should also be performed to obtain actual spinal sagittal balance.

\section{Conclusions}

Our results indicate significant interdependence between the spinopelvic and cervical alignment, especially in cervical symptomatic patients. In addition, strong correlations were found between the $\mathrm{C}_{7}$ SVA and $\mathrm{C}_{2} \mathrm{C}_{7}$ Cobb angle. Overall, the results of this study could help to better understand the cervical sagittal alignment and serve as preliminary data for planning surgical reconstruction procedures.

\section{Conflict of Interest}

No potential conflict of interest relevant to this article was reported.

\section{ORCID}

Babak Alijani: https://orcid.org/0000-0003-4480-6236 Javid Rasoulian: https://orcid.org/0000-0002-8389-0357

\section{References}

1. Kim D, Menger RP. Spine sagittal balance. StatPearls. Treasure Island (FL): StatPearls Publishing; 2019.

2. Schwab F, Ungar B, Blondel B, et al. Scoliosis Research Society-Schwab adult spinal deformity classification: a validation study. Spine (Phila Pa 1976) 2012;37:1077-82.

3. La Maida GA, Zottarelli L, Mineo GV, Misaggi B. Sagittal balance in adolescent idiopathic scoliosis: radiographic study of spino-pelvic compensation after surgery. Eur Spine J 2013;22 Suppl 6:S859-67.

4. Jang JS, Lee SH, Min JH, Kim SK, Han KM, Maeng DH. Surgical treatment of failed back surgery syndrome due to sagittal imbalance. Spine (Phila $\mathrm{Pa}$ 1976) 2007;32:3081-7.

5. Hiyama A, Katoh H, Sakai D, et al. Correlation analysis of sagittal alignment and skeletal muscle mass in patients with spinal degenerative disease. Sci Rep 
2018;8:15492.

6. Albert TJ, Vacarro A. Postlaminectomy kyphosis. Spine (Phila Pa 1976) 1998;23:2738-45.

7. Shimizu K, Nakamura M, Nishikawa Y, Hijikata S, Chiba K, Toyama Y. Spinal kyphosis causes demyelination and neuronal loss in the spinal cord: a new model of kyphotic deformity using juvenile Japanese small game fowls. Spine (Phila $\mathrm{Pa} 1976$ ) 2005;30:2388-92.

8. Schwab F, Farcy JP, Bridwell K, et al. A clinical impact classification of scoliosis in the adult. Spine (Phila Pa 1976) 2006;31:2109-14.

9. Manoharan SR, Joshi D, Owen M, Theiss SM, Deinlein D. Relationship of cervical sagittal vertical alignment after sagittal balance correction in adult spinal deformity: a retrospective radiographic study. Int J Spine Surg 2018;12:269-75.

10. Berthonnaud E, Dimnet J, Roussouly P, Labelle H. Analysis of the sagittal balance of the spine and pelvis using shape and orientation parameters. J Spinal Disord Tech 2005;18:40-7.

11. Scheer JK, Tang JA, Smith JS, et al. Cervical spine alignment, sagittal deformity, and clinical implications: a review. J Neurosurg Spine 2013;19:141-59.

12. Lee SH, Kim KT, Seo EM, Suk KS, Kwack YH, Son ES. The influence of thoracic inlet alignment on the craniocervical sagittal balance in asymptomatic adults. J Spinal Disord Tech 2012;25:E41-7.

13. Hasegawa K, Okamoto M, Hatsushikano S, et al. Standing sagittal alignment of the whole axial skeleton with reference to the gravity line in humans. J Anat 2017;230:619-30.
14. Nunez-Pereira S, Hitzl W, Bullmann V, Meier O, Koller H. Sagittal balance of the cervical spine: an analysis of occipitocervical and spinopelvic interdependence, with C-7 slope as a marker of cervical and spinopelvic alignment. J Neurosurg Spine 2015;23:16-23.

15. Guo Q, Ni B, Yang J, et al. Relation between alignments of upper and subaxial cervical spine: a radiological study. Arch Orthop Trauma Surg 2011;131:857-62.

16. Lee SH, Son ES, Seo EM, Suk KS, Kim KT. Factors determining cervical spine sagittal balance in asymptomatic adults: correlation with spinopelvic balance and thoracic inlet alignment. Spine J 2015;15:705-12.

17. Shao ZX, Yan YZ, Pan XX, et al. Factors associated with cervical spine alignment in an asymptomatic population: a preliminary analysis. World Neurosurg 2019;122:e48-58.

18. Matsubayashi Y, Chikuda H, Oshima Y, et al. C7 sagittal vertical axis is the determinant of the C5C7 angle in cervical sagittal alignment. Spine J 2017;17:622-6.

19. Lee CS, Chung SS, Kang KC, Park SJ, Shin SK. Normal patterns of sagittal alignment of the spine in young adults radiological analysis in a Korean population. Spine (Phila Pa 1976) 2011;36:E1648-54.

20. Singh R, Yadav SK, Sood S, Yadav RK, Rohilla R. Spino-pelvic radiological parameters in normal Indian population. SICOT J 2018;4:14.

21. Vrtovec T, Janssen MM, Likar B, Castelein RM, Viergever MA, Pernus F. A review of methods for evaluating the quantitative parameters of sagittal pelvic alignment. Spine J 2012;12:433-46. 